\title{
Benchmarking in mental health: an introduction for psychiatrists
}

\author{
Richard Bayney
}

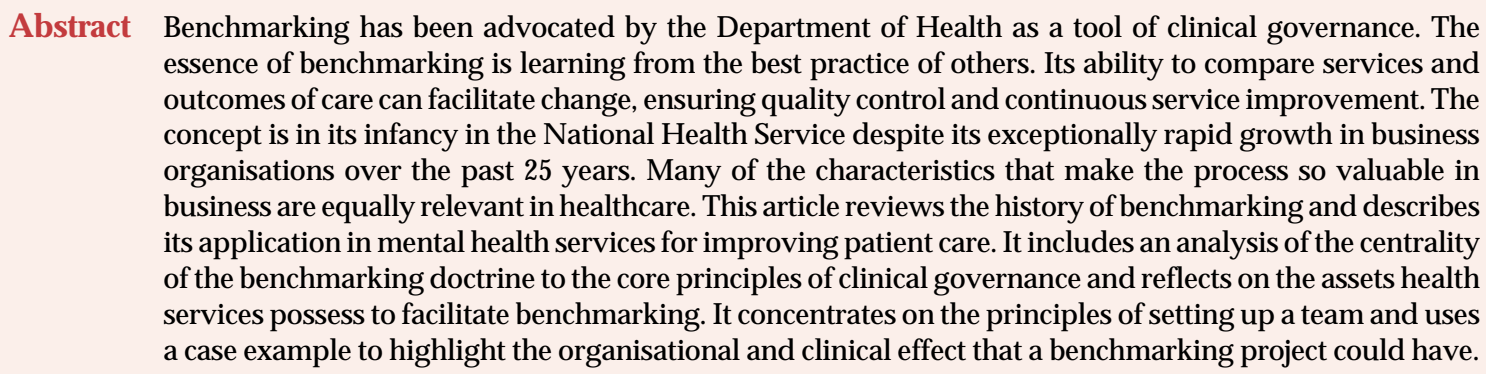

The concept of benchmarking was developed by the Xerox Corporation in 1979 and introduced into the North American corporate world in the 1980s. It continues to have a wide application as a business activity, mainly because it works. According to Camp \& Tweet (1994), the Japanese word dantotsu, which means striving to be the best of the best, captures the essence of benchmarking. In fact, the Japanese pursued benchmarking in several forms well before Xerox. In a relentless search for excellence, Japanese companies would lend employees to, or swap them with, other organisations. This practice encouraged employees to go outside their workplaces, assess their own internal business processes against others and return with new ideas, systems and processes to facilitate the development of their own organisations. However, it would be deceptively simple and potentially misleading to try to distil a process as complex as benchmarking to such an elementary notion.

A more formal definition, adapted from that of the Xerox Corporation itself, describes it as 'the continuous process of measuring products, services and practices against leaders, allowing the identification of best practices that will lead to measurable improvements in performance' (Camp, 1989). In the present context, benchmarking has little to do with setting benchmarks (i.e. providing measurement standards or references for others to meet or compare against), although some authorities still invoke this concept in their definition (Department of Trade and Industry, 2004). Nor is its full potential being exhausted when it is used as a method for producing guidelines or national standards for patient care (Bucknall et al, 2000). Benchmarking in the National Health Service (NHS) is evolving only slowly and is still used mostly to assess an organisation's position in relation to other services, with little analysis of the reasons for any gaps (Bullivant, 1996). This is certainly the case in mental health practice (McGowan et al, 1999; Mirza et al, 2003).

In an effort to make the concept more generalisable in other spheres, Robert Camp (1989) has reduced the definition to 'finding and implementing best practices'. This refinement alludes to a foundation that is not dissimilar to the framework underpinning evidence-based medicine, although the processes of evaluation of best practice are different. What is also different is the connotation surrounding the term 'best'. Whereas recently published evidence-based best practices for the treatment of schizophrenia (National Institute for Clinical Excellence, 2002) might be an established and shared concept across different mental health services, the best practice in the benchmarking sense for implementing that treatment varies, even between similar organisations, depending on their own unique situation. Thus, the custom within benchmarking is to learn from the best practice of others (the chosen 'partners') and to understand the processes by which performance can

Richard Bayney is a consultant psychiatrist at the Directorate of Forensic Psychiatry, West London Mental Health Care NHS Trust (Uxbridge Road, Southall, Middlesex UB1 3EU, UK. Tel: 0208354 8680; e-mail: Richard.Bayneyric@wlmht.nhs.uk). His clinical and research interests include the organisation and effectiveness of mental health services in the National Health Service. 
be enhanced, rather than simply to copy another process. In some circumstances, what is best for one organisation may be disastrous for another, and in this article I try to describe the intricacies and pitfalls associated with undertaking a benchmarking project. Several forms of benchmarking exist, and here I describe Camp's (1989) typology, which typically shows breakthrough results. Camp suggests that four types of benchmarking (internal, competitive, functional and generic process) should be carried out in the order listed below. Each has a specific outcome and benefit.

\section{Types of benchmarking Internal benchmarking}

Internal benchmarking should be the starting point of any benchmarking process. It is usually necessary to document internal working processes first and internal benchmarking is the most straightforward way of accomplishing this. The partner should be within another part of the service, not too geographically distant and should share similar functions and processes. For example, it would be relatively straightforward to compare appointment booking for out-patients in different hospital departments. The contrasting procedures may reveal some best practices within the organisation that can be modified and replicated elsewhere. Previous attempts at internal benchmarking have shown that practice varies greatly from area to area. This can be as simple as innovations in one ward being unappreciated on an adjacent ward (Patterson, 1993).

The advantages of internal benchmarking are numerous. As well as ease of data collection resulting from greater internal consistency, it is also relatively inexpensive and use of an internal partner can bring to light potential problems in working with external organisations. Another advantage is that it should not be difficult to find partners within the NHS: internal benchmarking is a natural choice for hospital systems, as there are multiple sites within a hospital and even multiple hospitals within a trust for comparison. It also allows sharing of comparative data and internal trends with departments within the service, allowing the exploration and integration of multidisciplinary approaches to optimise processes and outcomes. However, a limitation of internal benchmarking is that the level of the best performer within the organisation usually determines the level achieved by the rest.

More specialised services may consider their work to be too sophisticated for benchmarking because of the futility of finding a suitable partner. This is often a mistake, as it should be possible to concentrate on benchmarking of more general processes. For instance, most psychiatric services, including specialised services, have strategies for managing substance misuse. Benchmarking these shows what a partner is doing differently and perhaps more successfully.

\section{Competitive benchmarking}

The principal aim of competitive benchmarking is to compare a specific process with that of the best competitor in the same industry and to identify performance levels to be surpassed. This is the stage after baseline attempts at internal benchmarking. Competitive benchmarking is important because a progressive organisation, in order to assess its strengths and weaknesses, must at some stage assess the gap between its own operations and the competition. Occasionally, this process can be hindered if the competition is revealed to be performing less well than you are.

\section{Functional benchmarking}

Functions of an organisation that are performed in other industries as well as in healthcare form the basis of functional benchmarking. If the best partner operates within a different industry, the advantage of this type of comparison is that a mental health service attaining this level of benchmarking has the opportunity to improve functioning beyond the best NHS or non-NHS competitor. For example, a mental health service should find worth in benchmarking against institutions that have a reputation for consistent delivery of accurate and important information to staff, for example an airline service. Such an exercise could advance performance in shared similar functions, including providing customer satisfaction, information processing and risk management strategies. A disadvantage of functional benchmarking is that it does not focus on the processes of the partners. The lessons learnt might therefore be harder to implement because even though one is able to learn from the information processing function of an airline, transferring this knowledge to a mental health service requires considerable integration.

\section{Generic process benchmarking}

Generic process benchmarking allows benchmarking of specific processes across different industries to find the best practices wherever they may exist. For example, an ammunition manufacturer was able to produce smoother, shinier shells following consultation with a lipstick company. 
Senior hospital managers seeking to improve income might concentrate on increasing revenue from their hospital's assets by comparing against any company that is performing this process well. Sussex (1999) suggests that benchmarking against water and electricity companies should be enlightening even though at first glance they seem incompatible with health services. He highlights their similarities: for example they are local monopolies delivering essential services and they are affected by significant inherited inflexibilities such as location, capacity and aged equipment. Their many similar generic processes offer the hospital opportunity for a large improvement in performance.

\section{Outcomes}

Figure 1 illustrates the potential improvements in performance that each of the four types of benchmarking produces. Internal benchmarking offers the least potential for true breakthrough improvement, although it is a low-risk way to learn and practise the discipline. Navigating competitive and functional benchmarking requires more effort

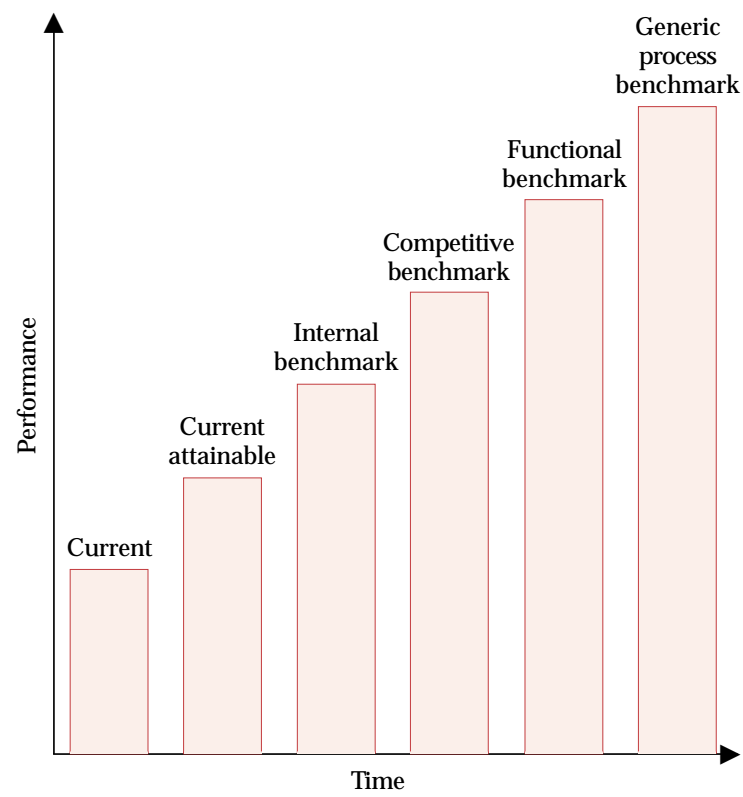

Fig. 1 Levels of attainment possible through benchmarking. Current attainable performance is that which could be realised by making optimal use of existing assets. For each of the other performance levels the degree of superior functioning will be related to the extent of the pursuit of best practice. Accomplishing the best performance will eventually entail benchmarking against institutions outside of healthcare. and resources, and their success depends on a greater familiarity with benchmarking methods. Generic process benchmarking is acknowledged as providing the best results, but is attendant on the organisation having matured through a series of projects and should not be undertaken by novice organisations without skilled guidance (Camp, 1989).

\section{Why should benchmarking be applied to healthcare? \\ Relationship to clinical governance}

Faced with yet another quality initiative the sceptical clinician may be wondering what advantages benchmarking offers. The Department of Health has challenged managers with the responsibility of commissioning and providing efficient service delivery through quality improvement activities (Department of Health, 1997). It has recommended benchmarking as an activity that, like audit, wellconsidered guidelines and other systematic reviews of practice, can inform the process of clinical governance (Department of Health, 1999). Clinical audit by itself has been recognised as having a poor record in improving practice (Hopkins, 1996). There is growing concern that the wealth of guidelines impinges on creativity, fails to solve the problems of poor care and may in some circumstances even be harmful (McDonald, 2003). Traditional measures of service use such as hospital readmission rates and length of stay often reflect service policy and provision in a self-fulfilling manner rather than giving true information about the impact of treatment on patients (Shooter, 1997). Psychiatrists also encounter difficulties in making sense of experimental evidence to inform clinical decision-making because of the small scale and short-term nature of most psychiatric trials (Wykes \& Marshall, 2004).

Benchmarking demonstrates several refinements over these other activities (Box 1), mainly because its doctrine of continuous improvement is a central tenet of clinical governance. It is a means by which the practices needed to reach new goals are discovered and understood. Benchmarking can be seen as a direction-setting procedure that helps to manage the relationship between systematic policy developments, inefficient processes, identified clinical pathways and evidence-based outcomes. It moves away from the traditional method of establishing targets, the extrapolation of internal past practices and trends. Benchmarking does not restrict an organisation to the limited supply of internal ideas and performance assessments advanced by initiatives such as quality circles (Cole, 1999). It also allows for potentially boundless 


\section{Box 1 Advantages of benchmarking}

Benchmarking has advantages over other quality initiatives because it is:

- practitioner led

- externally focused to broaden practitioners' horizons of what is realistically achievable

- research dependent

- evaluated by measurable and climate-focused outcomes

- audited locally but comparable widely

- realistic within the clinical setting

- able to emphasise the need to support a change

- supported by immediately available actionplanning

- able to recognise individuals' efforts

- able to prevent the waste of resources

- able to develop practice, not just monitor and sustain existing practice

- comparable with other systems of how best practice is achieved

- aiming for the best achievable anywhere, not just locally or even nationally

continuing improvement by comparison against a wealth of medical and business organisations anywhere in the world. Thus, a mental health service can keep up with the rapidly changing external environment and reduce staleness associated with conventional goal-setting.

It is important to recognise that effective benchmarking is not a one-off exercise. It is a continuing process of improvement with the expectation that as one exercise stops another should start. Box 2 illustrates some of the mechanisms by which benchmarking may improve practice. Naturally, there are limits to the improvements that can be achieved and, potentially, to the ability of an organisation to use benchmarking effectively.

\section{Limitations of benchmarking in mental health practice}

It might be argued that benchmarking is a valid exercise in mental health practice only if it produces an improvement in patient care. Use of a benchmarking team to improve an administrative or other support procedure is unsatisfcatory if there is no benefit for patient care. For example, significant expenditure on an industry-leading information technology system does not automatically lead to improved patient care (although that potential obviously exists). Worse, it may appropriate money from an existing service that was providing useful and appreciated support.
Box 2 Mechanisms by which benchmarking can improve practice in healthcare services

- Spurring on individual clinicians and whole units to be among the best may raise morale, which is important to patient care and good service delivery. This more positive approach may have greater success than did confrontational initiatives such as competition and contestability in the 1990s, which are widely perceived to have failed as stimuli for NHS trusts to provide services as efficiently as possible (Sussex, 1999).

- Benchmarking allows users and carers to be involved in the process of change, orienting services to meet changing user needs and focusing on key areas that require attention.

- Benchmarking is a natural tool for supporting and enhancing clinical governance by providing a constant drive to raise quality through its various types.

- It allows the NHS to measure its services in terms of results that are important to users. This may lead to the development of more appropriate measurement systems and establish realistic objectives that can be easily implemented.

- It creates a better understanding of the dynamics and practices of good health service performance and of other non-healthcare organisations.

- Healthcare workers may prefer working in an atmosphere that fosters growth, breakthrough thinking, innovation and high standards, qualities that in turn may lead to better staff retention and more consistent care of patients.

- Once identified, a best practice can be easily shared between members of the same or other health services, who can extract elements useful to them.

- Benchmarking can lead to reduced costs for an institution, enabling redistribution of money for other aspects of patient care.

- Systems theory dictates that incremental improvements to an existing system or process are less successful in achieving exponential performance improvement than is redesigning and replacing it. Benchmarking encourages just such fundamental change from outside the system, rather than relying on potentially limiting ideas for improvements from within it (Watzlawick et al, 1974). Changes to systems are more likely to occur with functional and generic process benchmarking than with internal benchmarking. 
Box 3 Inappropriate uses of benchmarking

- It is a poor use of resources for investigating matters of moderate-to-low impact

- It is a poor use of resources if it is only to be used as an information-gathering technique

- It should not simply copy the best practices of others

- It is not a form of competitive analysis

- It should not be seen as a pretext for visiting interesting companies

- It should not be viewed as providing a quick fix for problematic processes in psychiatric services. Even if an outstanding practice is found, it is likely that it will have to be modified in order for it to be made effective within one's own organisation

There are other factors that limit the ability of a mental healthcare organisation to use benchmarking effectively. First is the lack of good outcome measures for mental health services. Industrial companies can measure improvements in terms of reduced or improved profits, whereas mental health benchmarking outcomes are likely to be more qualitative and may require more careful deliberation. If it takes a long time to decide measurable outcomes before setting up a project or too long (more than about 6 months) to complete, a project a team can lose enthusiasm and support from within the organisation. The same can happen if healthcare services spend a lot of time in search of ideally compatible facilities against which to compare themselves rather than taking a broader approach and learning from wherever they can. Finally, initiating a project for the sake of undertaking benchmarking is usually much more difficult than starting one in an area where a specific breakthrough improvement has been long required (Mosel \& Gift, 1994).

Box 3 highlights some inappropriate uses of benchmarking.

\section{What assets do healthcare services have for benchmarking?}

Adapting benchmarking to the healthcare sector is no different in principle from adapting it to any other sector in which many different professionals with specialised functions work closely together. Camp \& Tweet (1994) suggest that most hospitals are relatively small organisations when compared with large industrial enterprises. This should make it easier for them to communicate internally, make rapid decisions, question a breadth of staff and access extensive records that contain much potentially valuable information (Lelliott, 2003). This has to be balanced against the likelihood that they will have fewer full-time clinical staff free to carry out a benchmarking project.

Internal and competitive benchmarking require a relatively open exchange of information. Although this may well benefit both parties in the long term, the benchmarking partner may be more reluctant than the 'petitioner' to reveal its processes. Fortunately, the health service involves organisations that are more ready to share information. For example, there is a wealth of readily available information about clinical risk management from audits, complaints, incident forms and inquiries within each hospital which is often limited to within a trust. Benchmarking of the processes by which other trusts use information about errors and complaints to augment performance should be relatively straightforward owing to the degree of candidness that health services cultivate. Benchmarking relies heavily on an atmosphere of trust, and such easy access to information may not be manifest when mental health services start benchmarking against non-medical companies, which may wish to protect their industrial secrets.

The following case example is fictional but faithfully reflects clinical reality. Any resemblance to an actual case is purely coincidental.

Case example

Dr S, a general adult psychiatrist working in St Cuthbert's district general hospital is dismayed by a complaint from the mother of a patient. Her son has been on a waiting list for cognitive-behavioural therapy (CBT) for psychosis for 10 months. The clinical director of the service, who has an interest in benchmarking, convinces Dr S to review why the waiting list is unacceptably long.

Dr S forms a team to map provision of CBT for psychosis (Fig. 2). It identifies a number of delays that generate an average waiting time of 13 months. For example, a questionnaire reveals that that there is only one psychologist available to provide one session a week, whereas the waiting list suggests a need for eight sessions a week. It also demonstrates that patients receive CBT for positive symptoms of schizophrenia but do not receive treatment for negative symptoms or poor social skills. The team members decide that, should they not find a better solution, they might use junior doctors and employ a part-time supervising psychologist to reduce the waiting list.

A serendipitous meeting with a manager in the primary care trust highlights a service recently set up in a large forensic psychiatric unit 10 miles away. It is within the same trust and appears to be an ideal internal benchmarking partner.

The team visit the unit, and find that the forensic service is using weekly group therapy to treat 31 patients divided between three groups. Treatment continues for 4 months, during which time it addresses social skills, and positive and negative symptoms. Six 


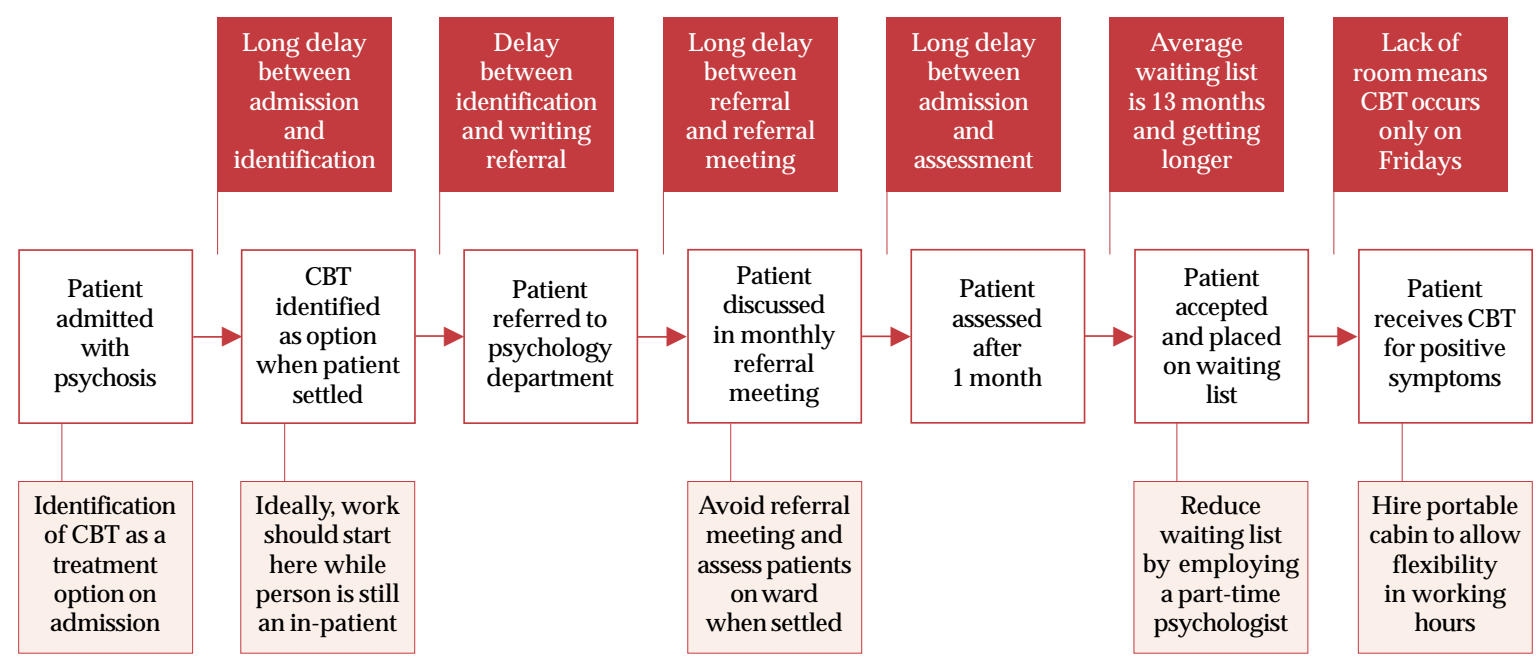

Stages of the in-patient journey common to many patients

Inefficiencies/blockages inhibiting access to CBT for patients with psychosis

Suggested solutions by the benchmarking team before they met the partners

Fig. 2 Process map of the pathway for an in-patient to receive CBT for psychosis

trained nurses and four trained psychologists run the groups. The six nurses also work on the wards and supervise other nurses. The groups are repeated three times per year and the average waiting time to join a group is 2 months.

The team members realise that they would never have devised such a scheme themselves. They adopt the practice in their own hosptial, providing similar groups on a smaller scale, and surpass their earlier goal.

The benchmarking exercise resolved the two most significant problems that had resulted in the previous sluggishness: the limited number of free rooms and a non-evidence-based belief that CBT had to be delivered individually.

\section{Conducting a study}

Many processes will appear suitable for benchmarking. It is recommended that people start with a small project that can be performed within 3-6 months, for which a benchmarking partner is easily identifiable and where objectives are specific and measurable. Preferably the project should result in improved patient care. It would be prudent to consider processes that users have said are inefficient, particularly one in which the team can identify a specific barrier to providing effective treatment for an identified need. Table 1 offers a variety of potential projects.

The next task is to identify key individuals to make up the benchmarking team, as outlined in Box 4.

\section{Analysis of internal practices}

Having established the process to be reviewed, the team should embark on an analysis of the current practices within it, using the tools of process mapping (Lenz et al, 1994). This groundwork allows the team to recognise steps crucial to meeting objectives, in addition to inefficient or redundant steps. Mapping is particularly useful in identifying blockages (e.g. the delays in Fig. 2), as well as augmenting any future comparison against external benchmarking partners when determining how they manage inefficiencies and impediments.

\section{Table 1 Potential benchmarking projects in mental health services}

\section{Aim}

Improving treatment of resistant schizophrenia Reducing average length of stay in seclusion Reducing prescription costs Improving cleaning of hospitals Provision of excellent food in hospitals Improving electronic record-keeping Improving security on site

\section{Benchmark}

An organisation that specialises in such treatment An organisation that does not have a seclusion room A service with a record of low costs

An organisation such as a large hotel chain An organisation such as a high-quality airline An organisation with a substantial electronic database An armed forces unit or prison 


\section{Box 4 Suggested key players in a benchmarking project}

The team leader

The benchmarking team

Functional experts

Mental health users and carers

The executive champion

The process sponsor

Stakeholders
Plans the development of projects and relays information to the whole organisation about the team's advancement

Team members undertake the majority of the project work and should ideally work with or be familiar with the process being benchmarked

Valued for their appreciation of how the service functions and ability to predict how change to one part of the process will ultimately affect the rest of the service

Add the customers' perspective, ensuring that the project does not result in ineffective change

Preferably a senior manager, not only taking responsibility for the project but also ensuring recognition of the team's work

A manager such as a clinical director with a direct interest in the results of the project and who has a closer involvement in the process than the executive champion

Although not involved in the benchmarking itself, their dissatisfaction with a service may help initiate the process; they are important in setting limits for the scope of the study and ensuring that the objectives of the study are met
It is advisable to conduct a pilot study before proceeding to the full study. This allows team members to familiarise themselves with the tools, modify their aims if necessary and, of paramount importance, encourages senior managers to support later work on the basis of positive results.

Choosing what to measure within a specific process requires identification of the essential quantifiable stages of the process that are aligned most closely with the service's duties, aims and objectives. The choice might be based on counting patient complaints about a process as a gauge of its success. If particular data cannot be used in achieving the specified improvement it is unlikely that they need to be gathered. For instance, user satisfaction is often regarded as a basic measure of service delivery. However, it may not be much use as a measure if the objective is to reduce out-patient waiting times, because it does not directly reflect any improvement in waiting times and is likely to be confounded by other variables.

Having identified the process and outcome measures, the next step is to confirm current levels of success, using a well-designed questionnaire. The first stage in designing the questionnaire is to agree an objective for it to test. The questionnaire should also be able to extract data from the benchmarking partner to discover the mechanisms behind their best practices. Questionnaire development is a complicated process, and an internal pilot questionnaire can help to rectify vagueness, eliminate jargon, ensure that the questionnaire asks the right questions and add to the data already gathered about the process in the organisation. Having as few questions as possible reduces the chance of alienating a benchmarking partner. Every question should contribute independently to realising the objectives of the study and not be repetitive or overlapping. Analysing the answers of the pilot will indicate how well the questionnaire meets these objectives.

\section{Identifying potential benchmarking partners and best practice}

In parallel with the above is the search for partners, often based on reputation for excellence, similarities of service and willingness to share data. The presentation to the partner needs to be intelligible, convincing and tested. Some of the key questions that help extract data from a partner are summarised in Box 5. Most of a team's time will be spent on collecting data rather than meeting with the benchmarking partner, and team members should not underestimate the amount of time required to grasp the intricacies of their information systems, extract information manually from records and pursue missing data. The procedure is simplified if there is a user-friendly system for retrieving data, some assurance that the data are accurate and the capability to amalgamate the best practices of one internal department with another.

Best practice is determined to a large extent by how much of an impact the practice has on the business, the degree to which the results are related to specific goals and whether the practice dovetails with other programmes and operations already being undertaken. For example, a system for dealing with service 
Box 5 Some questions to be considered when meeting with the benchmarking partner

With regard to the process under study:

- How do you define its success?

- What qualitative measures define the excellence of the process?

- What quantitative measures define the current performance of this process?

- What is the cost-effectiveness of the process?

- What training is provided for the different personnel involved in the process?

- Have any significant advances in operation been linked to particular improvements in the process?

- Is it possible to produce a map of the process?

- Is there any other information that might be helpful?

user complaints could be subjected to functional benchmarking against the customer complaints procedure of a successful manufacturer that receives relatively few complaints and manages these efficiently. The low level of complaints may be related to the manufacture of efficient and reliable products. Nevertheless, excellent integration of the manufacturing process with the practice of quick, satisfactory settlement of grievances leads to far superior performance, evidenced by even better sales.

\section{Analysing data and modifying practice}

Analysis of data should lead the team to ask whether there are pointless or inconsistent practices causing loss of direction or sluggish movement and whether each contributor to the process clearly understands the other participants' information requirements. For example, in a study optimising out-patient room use, it might be found that, compared with the partner, staff managing patient records in one part of the building do not know when clinicians require updated records in the clinic, or that the transport department underestimates the lead time necessary to convey patients punctually for their appointments. Knowing the cause of the discrepant practices between partners is only the beginning of the process because one then has to identify the factors that propel those differences, such as variations in the use of protocols between departments, unreliability of suppliers, antiquated facility design or uneasy interdepartmental dealings.

Modification of practice is likely to be beneficial and lead to better performance when differences and the reasons for those differences between partners have been identified.
Careful consideration is required in implementation of changes, as healthcare benchmarking is fundamentally different from its industrial equivalent. It is important to ensure that potential improvement does not take on a purely competitive nature. If it does, quality of patient care may be sacrificed for speed of service or cost savings. It is advisable to employ a technique such as the Plan, Do, Study, Act (PDSA) cycle (Langley et al, 1996), which provides a framework for testing whether planned changes actually make the desired improvements before they are fully implemented.

A mastery of benchmarking is related to an understanding of its key elements (Box 6). It also requires the team to understand the relationships between all individuals involved in the process and to promote a cohesive and creative way of working. Without regard to the needs of these individuals, any changes that are made may lead to increased tensions, fragmentation and discord. To avoid this, all findings must be communicated on a regular basis to the parties that will have to implement the changes. This regular feed of information often induces more commitment to change, as resistance typically stems from the fear of loss of control. Box 7 outlines some of the major aspects involved in managing change. Finally, any variation to processes should be made in collaboration with senior managers. Commitment from managers is vital to ensuring continuity, coordination, the involvement of relevant practitioners, removal of obstacles and access to sensitive information (Ellis, 1995). Without sufficient support or authority, the changes suggested are unlikely to be implemented or to be effective.

Box 6 The key elements of a successful benchmarking project

- A steering committee with an executive champion

- Guidance for the team members regarding the principles of benchmarking

- The support of senior management

- Pinpointing with users and staff the important processes to investigate

- Analysing the individual steps of the process

- Choosing an appropriate outcome indicator

- An analysis of internal functioning

- Pursuit of an appropriate partner

- Meeting the partner and analysing gaps in performance

- Modifying practice on the basis of the results of analysis

- Sharing the results of the project with all members of the organisation 
Box 7 The process of managing change within benchmarking

- Identify the organisational problem that needs to change

- Identify dissatisfaction with the present situation

- Identify the steps required to change it

- Identify exactly how it is going to impact on patient care

- Identify the potential costs of the change

- Consider the consequences of not changing

- Create the desire for the improvement by involving stakeholders, users and carers

- Enlist support from senior management

- Use a disciplined structured approach

\section{Conclusions}

The modernisation of the NHS requires the construction of frameworks for developing, testing and implementing changes that lead to improvement. This is still a major challenge. Benchmarking provides one such framework. It enables providers of health services to compare their operations against leading performers, to find and implement best practice and to continuously improve the quality of care provided to patients. However, it requires a disciplined and systematic approach and, perhaps more importantly, time and resources.

Would benchmarking benefit the clinician? All healthcare workers encounter inefficient and wasteful processes within their service or receive complaints about the services that they work alongside. Benchmarking offers a way of understanding why those processes have become poorer and provides a source of innovative solutions that have been tried and tested. Benchmarking is challenging and requires dynamism and endurance in an arena where time and enthusiasm are scarce resources. Inevitably, it is likely to be undertaken by those who reject compromise and who have a relentless aim to be the best.

\section{References}

Bucknall, C. E., Ryland. I., Cooper. A., et al (2000) National benchmarking as a support system for clinical governance. Journal of the Royal College of Physicians of London, 34, 5256.

Bullivant, J. R. N. (1996) Benchmarking in the UK National Health Service. International Journal of Health Care Quality Assurance, 9, 9-14.

Camp, R. C. (1989) Benchmarking: The Search for Industry Best Practice. New York: ASQC Press.

Camp, R. C. \& Tweet, A. G. (1994) Benchmarking applied to health care. Journal on Quality Improvement, 20, 229238.
Cole, R. E. (1999) Managing Quality Fads: How American Business Learned to Play the Quality Game. New York: Oxford Press.

Department of Health (1997) The New NHS: Modern, Dependable. London: HMSO.

Department of Health (1999) Clinical Governance: Quality in the New NHS (Health Service Circular 1999/065). London: HMSO.

Department of Trade and Industry (2004) Achieving Best Practice in Your Business. http://www.dti.gov.uk/ implementbestpractice/benchmarking.html

Ellis, J. (1995) Using benchmarking to improve practice. Nursing Standard, 9 (35) 24-30.

Hopkins, A. (1996) Clinical audit: time for a reappraisal? Journal of the Royal College of Physicians of London, 30, 415425.

Langley, G., Nolan, K., Nolan, T., et al (1996) The Improvement Guide: A Practical Approach to Enhancing Organisational Performance. San Francisco, CA: Jossey Bass Publishers.

Lelliott, P. (2003) Secondary uses of patient information. Advances in Psychiatric Treatment, 9, 221-228.

Lenz, S., Myers, S., Norlund. S., et al (1994) Benchmarking: finding ways to improve. Journal on Quality Improvement, 20, 250-259.

McDonald, P. (2003) Guidelines are not directives. Hospital Medicine, 64, 510-511.

McGowan, S., Wynaden, D., Harding, N., et al (1999) Staff confidence in dealing with aggressive patients: a benchmarking exercise. Australian and New Zealand Journal of Mental Health Nursing, 8, 104-108.

Mirza, I., Green, D. \& Luyombya, G. (2003) Benchmarking assertive community treatment: a report from Waltham Forest. Clinical Governance: An International Journal, 8, 218221

Mosel. D. \& Gift, B. (1994) Collaborative benchmarking in health care. Journal on Quality Improvement, 20, 239249

National Institute for Clinical Excellence (2002) Guidance on the Use of Newer (Atypical) Antipsychotics for the Treatment of Schizophrenia (Health Technology Appraisal 43). London: NICE.

Patterson, P. (1993) Benchmarking study identifies hospitals' best practices. OR Manager, 9 (4) 11-15.

Shooter, M. (1997) What a patient can expect from a consultant psychiatrist. Advances in Psychiatric Treatment, $3,119-125$.

Sussex, J. (1999) Cost benchmarking - utilities show the way. British Journal of Healthcare Management, 5, 265-267.

Watzlawick, P., Wealdand, J. H. \& Fisch, R. (1974) Change: Principles of Problem Formation and Problem Resolution. Norton: New York.

Wykes, T. \& Marshall, M. (2004) Reshaping mental health practice with evidence: the Mental Health Research Network. Psychiatric Bulletin, 28, 153-155.

\section{MCQs}

1 Benchmarking of a process:

$\mathrm{a}$ is the designing and implementing of benchmarks

b may assist in uncovering the worst practices

c may be undertaken with very little resources

d should be completed within 180 days

e is a simple information-collecting technique.

2 Benchmarking is impeded by:

a staff awareness of the process being studied

b lack of support for the project at each level of the organisation

c difficulty defining suitable outcome indicators

$\mathrm{d}$ the sharing of information with other organisations

e a wealth of suitable partners. 
3 Of Camp's four types of benchmarking, internal benchmarking:

a should not be attempted first

$\mathrm{b}$ is difficult to accomplish within healthcare settings

c allows one to compare against the best in the world

$\mathrm{d}$ is easier because there are fewer barriers to data collection

e may result in the collection of benchmarks.

4 Benchmarking has advantages over other quality initiatives because:

a the results of a benchmarking project are usually easily adapted within an organisation

$b$ it seeks to raise standards of a service to above the average c in seeking best practice it is not limited to looking within the health service

$\mathrm{d}$ an identified best practice can be used by any number of similar organisations

e it identifies innovative solutions that have already been tried and tested.

5 The development of a good benchmarking project is helped by:

a the setting up of a benchmarking team

$b$ choosing a project that can be performed at a slow pace

c considering projects that users have identified as being beneficial

d prior work on a pilot study

e finding a partner against which to benchmark.

\section{Launching APT eLetters}

Aggravated by an article in APT? - Now you can tell us!

You can now comment immediately on APT's content.

The new eLetters section featured in the online APT is a quick and easy method for you to submit a rapid response to any article.

Accepted letters are posted online within 7 days of your submitting them. The Editor moderates all correspondence before it is posted on the site, where it can be accessed through a general link or directly from the article to which it refers. All eLetters are considered for subsequent publication in the print correspondence column.

Try for yourself from an online full-text article.

http://apt.rcpsych.org

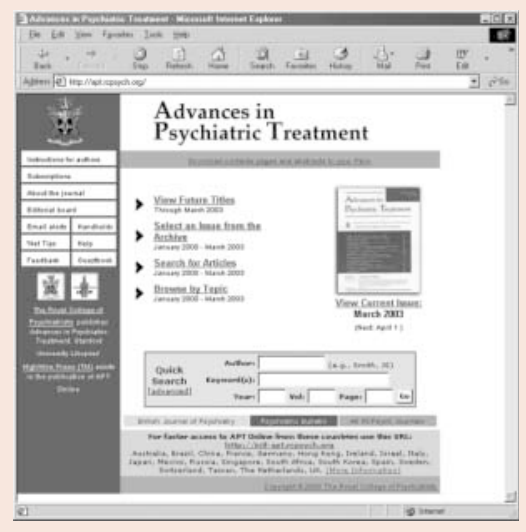

